\title{
Variational reduced density matrix method in the doubly occupied configuration interaction space using three-particle $\mathrm{N}$-representability conditions
}

\author{
Diego R. Alcoba, ${ }^{1,2, a)}$ Pablo Capuzzi, ${ }^{1,2}$ Alvaro Rubio-García, ${ }^{3}$ Jorge Dukelsky, ${ }^{3}$ \\ Gustavo E. Massaccesi, ${ }^{4}$ Ofelia B. Oña, ${ }^{5}$ Alicia Torre,${ }^{6}$ and Luis Lain ${ }^{6}$ \\ ${ }^{1}$ Departamento de Física, Facultad de Ciencias Exactas y Naturales, Universidad de Buenos Aires, \\ Ciudad Universitaria, 1428 Buenos Aires, Argentina \\ ${ }^{2}$ Instituto de Física de Buenos Aires, Consejo Nacional de Investigaciones Científicas y Técnicas, \\ Ciudad Universitaria, 1428 Buenos Aires, Argentina \\ ${ }^{3}$ Instituto de Estructura de la Materia, CSIC, Serrano 123, 28006 Madrid, Spain \\ ${ }^{4}$ Departamento de Ciencias Exactas, Ciclo Básico Común, Universidad de Buenos Aires, \\ Ciudad Universitaria, 1428 Buenos Aires, Argentina \\ ${ }^{5}$ Instituto de Investigaciones Fisicoquímicas Teóricas y Aplicadas, Universidad Nacional \\ de La Plata, CCT La Plata, Consejo Nacional de Investigaciones Científicas y Técnicas, \\ Diag. 113 y 64 (S/N), Sucursal 4, CC 16, 1900 La Plata, Argentina \\ ${ }^{6}$ Departamento de Química Física, Facultad de Ciencia y Tecnología, Universidad del País Vasco, \\ Apdo. 644, E-48080 Bilbao, Spain
}

(Received 13 September 2018; accepted 31 October 2018; published online 20 November 2018)

\begin{abstract}
Ground-state energies and two-particle reduced density matrices (2-RDMs) corresponding to $\mathrm{N}$-particle systems are computed variationally within the doubly occupied configuration interaction (DOCI) space by constraining the 2-RDM to satisfy a complete set of three-particle $N$-representability conditions known as three-positivity conditions. These conditions are derived and implemented in the variational calculation of the 2-RDM with standard semidefinite programming algorithms. Ground state energies and 2-RDMs are computed for $\mathrm{N}_{2}, \mathrm{CO}, \mathrm{CN}^{-}$, and $\mathrm{NO}^{+}$molecules at both equilibrium and nonequilibrium geometries as well as for pairing models at different repulsive interaction strengths. The results from the full three-positivity conditions are compared with those from the exact DOCI method and with approximated 2-RDM variational ones obtained within two-positivity and two-positivity plus a subset of three-positivity conditions, as recently reported [D. R. Alcoba et al., J. Chem. Phys. 148, 024105 (2018) and A. Rubio-García et al., J. Chem. Theory Comput. 14, 4183 (2018)]. The accuracy of these numerical determinations and their low computational cost demonstrate the usefulness of the three-particle variational constraints within the DOCI framework. Published by AIP Publishing. https://doi.org/10.1063/1.5056247
\end{abstract}

\section{INTRODUCTION}

The direct solution of the $N$-particle Schrödinger equation is impractical except for the smallest systems as the cost of solving a three-dimensional partial differential equation for $N$ particles to a given accuracy $\chi$ typically grows exponentially, as $\chi^{N}$. This is known as the exponential wall problem. ${ }^{1}$ Over the years, many approximations have been applied to the full configuration interaction (FCI) calculation to reduce the computational cost so that dealing with larger systems becomes feasible. One such approximation is the doubly occupied configuration interaction (DOCI) method $^{2-10}$ where the configuration interaction Hilbert space is restricted to doubly occupied determinants. The DOCI method is able to suitably describe strongly correlated systems, capturing most of the static correlation, and serving as the first step on expansions approaching to the exact FCI solution. ${ }^{8,11-15}$

a)Author to whom correspondence should be addressed: qfxaldad@lg.ehu.es
Despite the restriction imposed in the configuration interaction space, the DOCI method still scales exponentially with the system size. For a single-particle basis set of $K$ orbitals, a DOCI $N$-electron wave function is expanded on $\left(\begin{array}{c}K \\ \frac{N}{2}\end{array}\right) N$-electron Slater determinants, while a FCI wave function requires $\left(\begin{array}{l}K \\ \frac{N}{2}\end{array}\right) \cdot\left(\begin{array}{c}K \\ \frac{N}{2}\end{array}\right) N$-electron ones. However, the non-polynomial scaling can be avoided since the $N$-particle wave function contains more information than that necessary for computing the energy as the knowledge of the two-particle reduced density matrix (2-RDM) alone suffices. ${ }^{16-22}$ Nevertheless, direct optimization of the energy with respect to the 2-RDM is challenging because not every 2-RDM stems from an N-particle wave function or density matrix. ${ }^{20,23,24}$ To ensure that the 2RDM actually derives from a physical state, a set of constraints on the 2-RDM, known as $N$-representability conditions, ${ }^{24}$ must be imposed. The necessary and sufficient conditions are known, ${ }^{25-28}$ but in practice, only a limited set of such constrains is used. Usually, the variational 2-RDM (v2RDM) calculations use a set of necessary $N$-representability constraints 
involving $p$-particle RDMs ( $p$-RDM), known as $p$-positivity conditions. ${ }^{28-30}$ In the DOCI space, recent calculations with molecular systems $\mathrm{s}^{31-33}$ and pairing models $\mathrm{s}^{34-36}$ have shown that the lower bounds on the ground-state energies obtained from v2RDM calculations converge rapidly with the number $p$ of particles. Moreover, for attractive pairing interactions twopositivity conditions together with a subset of three-positivity conditions have shown to provide exact numerical results for a large variety of integrable models. ${ }^{35}$ However, repulsive pairing models, more adequate to simulate the electronic interaction in molecular systems, have not been yet exploited as benchmarks of the theory. Despite the promising calculations and the accuracy of two- and partial three-positivity conditions, to our knowledge, the full three-positivity conditions comprising four types of three-particle probability distributions have not been yet implemented within the DOCI space.

In this paper, the full three-positivity conditions are developed and applied in variational 2-RDM calculations to manyelectron molecules and integrable pairing models. To this end, we derive and implement these $N$-representability conditions in the DOCI space, relating them to the two- and partial three-representability conditions already considered. The minimization of the energy with respect to a 2-RDM constrained by these positivity conditions constitutes a special optimization problem known as semidefinite programming. We report numerical calculations performed with the semidefinite programming algorithm (SDPA) described in Refs. 37 and 38 for the ground-state energies and 2-RDMs in the DOCI space for several molecules at both equilibrium and nonequilibrium geometries and for integrable pairing models with repulsive interactions. These results have been compared with those arising from DOCI calculations, showing that the employed threepositivity conditions provide a significant improvement upon those obtained using only two- and partial three-positivity conditions, while retaining the same computational scaling. Moreover, since the DOCI results are not invariant under a unitary orbital transformation, $, 8,11,31,39,40$ we have performed studies on the basis set dependence of our results.

This work is organized as follows. In Sec. II, we review the foundations of the variational reduced density matrix theory and introduce the equations which formulate the full threepositivity $N$-representability conditions in the DOCI space. Section III presents the implementation and the first applications of our theory. The computational details are given in Sec. III A, while the results obtained for selected molecular systems with several orbital basis sets are given in Sec. III $\mathrm{B}$, and those for repulsive pairing models in Sec. III C. Finally, in Sec. IV, we summarize the main conclusions of this work.

\section{THEORY}

The variational reduced density matrix theory and its formulation in the doubly occupied configuration interaction space are discussed in Secs. II A and II B, respectively.

\section{A. Variational reduced density matrix theory}

In second quantization, an $N$-particle Hamiltonian with pairwise interactions can be written as ${ }^{41}$

$$
H=\sum_{i j} t_{i j} a_{i}^{\dagger} a_{j}+\frac{1}{4} \sum_{i j k l} V_{i j k l} a_{i}^{\dagger} a_{j}^{\dagger} a_{l} a_{k},
$$

where $t_{i j}$ and $V_{i j k l}$ are the one-body energy and the twobody interaction terms, respectively. $a_{i}^{\dagger}$ and $a_{j}$ are the standard fermion creation and annihilation operators in a given orthonormal single-particle basis $\{i, j, k, l, \ldots\}$.

The ground-state energy can be expressed solely in terms of the second-order reduced density matrix, 2-RDM, ${ }^{2} D$ as $^{16-18}$

$$
E_{0}=\frac{1}{4} \sum_{i j k l} H_{i j k l}^{(2)}{ }^{2} D_{i j k l},
$$

where

$$
{ }^{2} D_{i j k l}=\left\langle\psi\left|a_{i}^{\dagger} a_{j}^{\dagger} a_{l} a_{k}\right| \psi\right\rangle
$$

and

$$
H_{i j k l}^{(2)}=\frac{1}{N-1}\left(t_{i k} \delta_{j l}-t_{j k} \delta_{i l}-t_{i l} \delta_{j k}+t_{j l} \delta_{i k}\right)+V_{i j k l}
$$

is the two-particle reduced Hamiltonian with $|\psi\rangle$ being the ground-state wave function and $N$ being the number of particles.

While Eq. (2) indicates that the ground-state energy of an $N$-particle system may be computed by direct variation of ${ }^{2} D$, this is not the case because not every 2-RDM can be obtained from the integration of an $N$-particle wave function. ${ }^{20,24}$ In general, a hierarchy of necessary, albeit not sufficient, set of constraints on the $p$-RDM are the $p$-positivity $N$-representability conditions. ${ }^{30,42}$ These conditions could be derived from the positive semidefinite property of a class of non-negative Hamiltonians of the form

$$
H=B^{\dagger} B
$$

where $B$ is a $p$-particle operator. As its expectation value must be non-negative, its matrix representation must be positive semidefinite. Different forms of this operator lead to different $p$-positivity conditions. In the two-particle space, these yield the well-known $2-\mathcal{P}, 2-\mathcal{Q}$, and $2-\mathcal{G}$ two-positivity conditions, ${ }^{24,25}$ which require the positive semidefiniteness of the two-particle, two-hole, and particle-hole reduced density matrices, ${ }^{2} D,{ }^{2} Q$, and ${ }^{2} G$, respectively, whose matrix elements are defined by Eq. (3) and

$$
\begin{aligned}
& { }^{2} Q_{i j k l}=\left\langle\psi\left|a_{i} a_{j} a_{l}^{\dagger} a_{k}^{\dagger}\right| \psi\right\rangle, \\
& { }^{2} G_{i j k l}=\left\langle\psi\left|a_{i}^{\dagger} a_{j} a_{l}^{\dagger} a_{k}\right| \psi\right\rangle .
\end{aligned}
$$

Even though these three representations of the 2-RDM can be related to each other by rearranging the creation and annihilation operators, restricting any of these matrices to be positive semidefinite does not imply that the other two matrices are positive semidefinite as well. Therefore, each of the twopositivity conditions furnishes distinct $N$-representability constraints of the 2-RDM. Because the two-particle metric matrices Eqs. (3), (6), and (7) can be obtained from three-particle metric matrices by contraction, the two-positivity conditions are implied by the three-positivity ones, also known as the $3-\mathcal{P}, 3-\mathcal{Q}, 3-\mathcal{E}$, and $3-\mathcal{F}$ conditions, ${ }^{30,43-45}$ which require that the following matrix elements constitute positive semidefinite matrices: 


$$
\begin{aligned}
& { }^{3} D_{i j k n m l}=\left\langle\psi\left|a_{i}^{\dagger} a_{j}^{\dagger} a_{k}^{\dagger} a_{l} a_{m} a_{n}\right| \psi\right\rangle, \\
& { }^{3} Q_{i j k n m l}=\left\langle\psi\left|a_{i} a_{j} a_{k} a_{l}^{\dagger} a_{m}^{\dagger} a_{n}^{\dagger}\right| \psi\right\rangle, \\
& { }^{3} E_{i j k n m l}=\left\langle\psi\left|a_{i}^{\dagger} a_{j}^{\dagger} a_{k} a_{l}^{\dagger} a_{m} a_{n}\right| \psi\right\rangle, \\
& { }^{3} F_{i j k n m l}=\left\langle\psi\left|a_{i} a_{j} a_{k}^{\dagger} a_{l} a_{m}^{\dagger} a_{n}^{\dagger}\right| \psi\right\rangle .
\end{aligned}
$$

Analogously to the two-positivity conditions, each of these three-positivity conditions provides distinct constraints on the $\mathrm{N}$-representability of the 3-RDM and, by contraction, of the 2-RDM. A subset of the three-positivity conditions, known as the $\mathcal{T}_{1}$ and $\mathcal{T}_{2}$ constraints, ${ }^{46-48}$ can also be defined as the positive semidefiniteness of the following combination of metric matrices:

$$
\begin{aligned}
& T_{1}={ }^{3} D+{ }^{3} Q, \\
& T_{2}={ }^{3} E+{ }^{3} F .
\end{aligned}
$$

These matrices are expressible in terms of the 2-RDM only because the terms with six creation and/or annihilation operators in the three-particle metric matrices cancel upon addition due to opposite signs. Note that due to their linear relation, the positivity conditions on ${ }^{3} D,{ }^{3} Q,{ }^{3} E$, and ${ }^{3} F$ warrant the positivity of $T_{1}$ and $T_{2}$; however, the converse is not true.

The ground-state energy can be minimized with respect to the 2-RDM subject to these conditions by a family of optimization techniques known as semidefinite programming. ${ }^{49-52}$ However, as these conditions are necessary but not sufficient, and thus the selected Hilbert space is larger than the physical one, the variational 2-RDM method will always find a lower bound to the exact ground-state energy and an approximation to the exact ground-state 2-RDM.

\section{B. Variational reduced density matrix theory in the $\mathrm{DOCl}$ space}

Here we will focus on Hamiltonians with interactions in the seniority-zero subspace, i.e., interactions that do not change the number of paired particles. Assuming time-reversal symmetry, the single-particle levels are doubly degenerate in the spin degree of freedom. The seniority quantum number classifies the Hilbert space into subspaces with a given number of singly occupied levels. ${ }^{53}$ The most general Hamiltonian within the seniority-zero subspace is

$$
H=\sum_{i} \epsilon_{i} n_{i}+\sum_{i \neq j} w_{i j} n_{i} n_{j}+\sum_{i j} v_{i j} b_{i}^{\dagger} b_{j},
$$

where $\epsilon_{i}$ are the energies of $K$ doubly degenerate singleparticle levels, $w_{i j}$ is the monopole interaction, and $v_{i j}$ is the pairing interaction. The number operator is $n_{i}=\frac{1}{2}\left(a_{i}^{\dagger} a_{i}+a_{\bar{i}}^{\dagger} a_{\bar{i}}\right)$, and the pair-creation (annihilation) operators are $b_{i}^{\dagger}=\left(b_{i}\right)^{\dagger}$ $=a_{i}^{\dagger} a_{\bar{i}}^{\dagger}$. The $(i, \bar{i})$ pair defines the pairing scheme, which can involve two particles with either opposite spins $(i \uparrow, i \downarrow)$, momenta $(i,-i)$, or in general any classification of conjugate quantum numbers in doubly degenerate single-particle levels. The DOCI Hamiltonian (14) is based on the SU(2) pair algebra with generators $b_{i}^{\dagger}$ and $\left(2 n_{i}-1\right) / 2$, and hard-core boson relations

$$
\left[b_{i}, b_{j}^{\dagger}\right]=\delta_{i j}\left(1-2 n_{i}\right), \quad\left(b_{i}^{\dagger}\right)^{2}=0 .
$$

For this Hamiltonian, the seniority number is also an exact quantum number, as unpaired particles do not interact with the rest of the system and the pairing Hamiltonian does not allow for pair breaking. The Hamiltonian thus becomes block diagonal in sectors labeled by the seniority quantum number.

The ground-state energy can be cast as $5,31,54$

$$
E_{0}=\sum_{i j} J_{i j} \Pi_{i j}+\sum_{i j} w_{i j} D_{i j},
$$

where

$$
J_{i j}=\delta_{i j} \epsilon_{i}+v_{i j} .
$$

The П matrix

$$
\Pi_{i j}=\left\langle\psi\left|b_{i}^{\dagger} b_{j}\right| \psi\right\rangle
$$

together with the $D$ matrix

$$
D_{i j}=\left\langle\psi\left|n_{i} n_{j}\right| \psi\right\rangle, \quad \forall i \neq j, \quad \text { and } \quad D_{i i}=\Pi_{i i}
$$

define the seniority blocks of the 2-RDM. According to these definitions, it follows that the $\Pi$ and $D$ matrices are Hermitian and fulfill

$$
\begin{gathered}
\sum_{i} \Pi_{i i}=\sum_{i} D_{i i}=M, \\
\sum_{j} D_{i j}=M \Pi_{i i},
\end{gathered}
$$

where $M$ is the number of particle pairs in the studied system. Note that the diagonal elements of both matrices are equal to the 1-RDM ones, $\rho_{i}=\left\langle\psi\left|n_{i}\right| \psi\right\rangle=\Pi_{i i}=D_{i i}$. The two- and partial three-positivity conditions can thus be written in terms of the seniority blocks of the 2-RDM as previously shown by Weinhold and Wilson ${ }^{5,54}$ and others. ${ }^{15,31,32,35,39}$ Here we refer the reader to the Appendix for the complete expressions.

In the seniority-zero subspace, the full three-positivity conditions can be expressed in terms of the two seniority blocks of the 3-RDM

$$
\begin{aligned}
D_{i j k} & =\left\langle\psi\left|n_{i} n_{j} n_{k}\right| \psi\right\rangle, \quad \forall i \neq j \neq k \\
\Pi_{j k}^{i} & =\left\langle\psi\left|b_{j}^{\dagger} n_{i} b_{k}\right| \psi\right\rangle, \quad \forall i \neq j, k,
\end{aligned}
$$

which are Hermitian and relate to the 1- and 2-RDM counterparts as

$$
\begin{gathered}
\rho_{i}=\frac{1}{M-1}\left(\sum_{j<i} \Pi_{i i}^{j}+\sum_{i>j} \Pi_{j j}^{i}\right), \quad \forall i, \\
\Pi_{i j}=\frac{1}{M-1} \sum_{k \neq i j} \Pi_{i j}^{k}, \quad \forall i<j, \\
D_{i j}=\Pi_{j j}^{i}, \quad \forall i<j, \\
\Pi_{j j}^{i}=\frac{1}{M-2} \sum_{k \neq i j} D_{i j k}, \quad \forall i<j .
\end{gathered}
$$

According to these definitions, the three-positivity conditions are obtained from the semidefiniteness of the four representations of the 3-RDM. These conditions were partially formulated in Refs. 5 and 54 and extended further more recently. ${ }^{32,39}$ The full set of constraints are as follows: 
- The 3-P condition: from the three-particle representation of the 3-RDM, we obtain

$$
\begin{aligned}
D_{i j k} & \geq 0, \quad \forall i<j<k, \\
\Pi^{a} & \geq 0, \quad \forall a .
\end{aligned}
$$

- The 3- $\mathcal{Q}$ condition: similarly, the three-hole 3-RDM gives rise to

$$
\begin{gathered}
1-\rho_{i}-\rho_{j}-\rho_{k}+D_{i j}+D_{j k}+D_{k i}-D_{i j k} \geq 0, \quad \forall i<j<k, \\
\mathcal{Q}^{a} \geq 0, \quad \forall a,
\end{gathered}
$$

with

$$
\mathcal{Q}_{i j}^{a}=-\Pi_{i j}^{a}+\Pi_{j i}+\delta_{i j}\left(1-2 \rho_{i}-\rho_{a}+2 D_{i a}\right), \quad \forall i j \neq a .
$$

- The 3-E condition: from the mixed two-particle-one-hole 3-RDM

$$
\begin{gathered}
\mathcal{E}_{a b c}=\left(\begin{array}{ccc}
D_{a b}-D_{a b c} & \Pi_{b c}^{a} & \Pi_{a c}^{b} \\
\Pi_{b c}^{a} & D_{a c}-D_{a b c} & \Pi_{a b}^{c} \\
\Pi_{a c}^{b} & \Pi_{a b}^{c} & D_{b c}-D_{a b c}
\end{array}\right) \geq 0, \quad \forall a<b<c, \\
\mathcal{E}_{a}=\left(\begin{array}{ccc}
D_{a i j}+\delta_{i j} D_{a i} & \Pi_{a j}^{i} & D_{i a} \\
\Pi_{a i}^{j} & \Pi_{i j}-\Pi_{i j}^{a} & \Pi_{i a} \\
D_{a i} & \Pi_{a i} & \rho_{a}
\end{array}\right) \geq 0, \quad \forall a, i j \neq a .
\end{gathered}
$$

- The 3-F $\mathcal{F}$ condition: from the mixed one-particle-two-holes 3-RDM

$$
\begin{gathered}
\mathcal{F}_{a b c}=\left(\begin{array}{ccc}
\rho_{a}-D_{a b}-D_{a c}+D_{a b c} & \Pi_{a c}-\Pi_{a c}^{b} & \Pi_{a b}-\Pi_{a b}^{c} \\
\Pi_{a c}-\Pi_{a c}^{b} & \rho_{c}-D_{b c}-D_{a c}+D_{a b c} & \Pi_{b c}-\Pi_{b c}^{a} \\
\Pi_{a b}-\Pi_{a b}^{c} & \Pi_{b c}-\Pi_{b c}^{a} & \rho_{b}-D_{a b}-D_{b c}+D_{a b c}
\end{array}\right) \geq 0, \quad \forall a<b<c, \\
\mathcal{F}_{a}=\left(\begin{array}{ccc}
-D_{a i j}+\delta_{i j}\left(\rho_{i}-D_{a i}\right)+D_{i j} & -\Pi_{a j}^{i}-\delta_{i j} \Pi_{i a} & \rho_{i}-D_{i a} \\
-\Pi_{a j}^{i}-\delta_{i j} \Pi_{i a} & \Pi_{i j}^{a}+\delta_{i j}\left(\rho_{a}-2 D_{i a}\right) & -\Pi_{i a} \\
\rho_{i}-D_{a i} & -\Pi_{a i} & 1-\rho_{a}
\end{array}\right) \geq 0, \quad \forall a, i j \neq a .
\end{gathered}
$$

\section{APPLICATIONS}

In this section, we will discuss our computational methodology followed by applications to molecular systems and pairing models in Secs. III B and III C.

\section{A. Computational details}

The variational optimization of the 2-RDM in the DOCI space was performed for three sets of $N$-representability conditions: the two-positivity (2-POS) conditions, the two-positivity plus the $\mathcal{T}_{1}$ and $\mathcal{T}_{2}$ partial three-positivity [(2,3)-POS] conditions, and the full three-positivity (3-POS) conditions. This optimization has been formulated as a semidefinite problem (SDP) in which the energy, being a linear function of the 2$\mathrm{RDM}$, is minimized over the intersection of a linear affine space and the convex cone of block-diagonal positive semidefinite matrices. ${ }^{49-52}$ We have developed codes that allow to efficiently formulate and solve the SDP exploiting the sparse structure of the matrices from the three sets of $p$-positivity conditions induced by the structure of the seniority-zero wave functions. Thus, the SDP in the seniority-zero subspace computationally scales with the number $K$ of single-particle basis elements as $O\left(K^{3}\right)$ for the 2-POS conditions and as $O\left(K^{4}\right)$ for both the (2,3)-POS and the full 3-POS conditions. This scaling is due to the sparse structure of the three-particle metric matrices within the DOCI framework, which possess $O\left(K^{3}\right)$ blocks of $O(1 \times 1)$ dimension and $O(K)$ blocks of $O(K \times K)$ dimension, in contrast to the sparse structure of the two-particle ones which present $O\left(K^{2}\right)$ blocks of $O(1 \times 1)$ dimension and $O(1)$ blocks of $O(K \times K)$ dimension. This will allow us to treat without excessive computational efforts systems of sizes up to $K=40$. In our numerical calculations, we use the semidefinite programming algorithm (SDPA) code. ${ }^{37,38}$ This code solves semidefinite problems at several precision levels by means of the Mehrotra-type predictor-corrector primaldual interior-point method, providing ground-state energies and the corresponding 2-RDMs. We programmed the variational reduced density matrix method as a dual problem in the SDPA code, which does not allow for the equality constraints Eqs. (20), (21), and (24)-(27). These are included by relaxing them into inequality constraints with a sufficiently small summation error $\delta .{ }^{47,55}$ In our work, we have set $\delta=10^{-7}$, which effectively fixes the precision of the groundstate energies.

\section{B. Molecular systems} by For molecular systems, the integrals in Eq. (1) are given 
TABLE I. Non-parallelity (NPE) and Maximum Absolute (MAE) errors of the ground state potential energy curves of molecules and ions calculated by the v2RDM-DOCI method imposing the 2-POS, (2,3)-POS, and 3-POS conditions from the DOCI results using the canonical molecular orbitals (CMO), natural orbitals (NO), orbitals which minimize the seniority number of the FCI wave functions $\left(\mathrm{M}_{\min }\right)$, and orbitals which minimize the DOCI energy (OPTE). Curves are computed in the interval $[1.4,4.0]$ bohr. Results correspond to standard STO-3G atomic basis set. The 2-POS and (2,3)-POS data have already been described in Ref. 33.

\begin{tabular}{|c|c|c|c|c|c|c|c|}
\hline & & \multicolumn{3}{|c|}{$\operatorname{MAE}\left(E_{h}\right)$} & \multicolumn{3}{|c|}{$\operatorname{NPE}\left(E_{h}\right)$} \\
\hline & & 2-POS & $(2,3)-\mathrm{POS}$ & 3-POS & 2-POS & $(2,3)-\mathrm{POS}$ & 3-POS \\
\hline \multirow{4}{*}{$\mathrm{N}_{2}$} & $\mathrm{CMO}$ & $1.731 \times 10^{-3}$ & $3.804 \times 10^{-5}$ & $1.055 \times 10^{-5}$ & $1.649 \times 10^{-3}$ & $2.614 \times 10^{-5}$ & $4.828 \times 10^{-6}$ \\
\hline & NO & $1.088 \times 10^{-3}$ & $2.523 \times 10^{-5}$ & $9.920 \times 10^{-6}$ & $9.801 \times 10^{-4}$ & $1.327 \times 10^{-5}$ & $5.008 \times 10^{-6}$ \\
\hline & $\mathrm{M}_{\min }$ & $1.137 \times 10^{-3}$ & $2.661 \times 10^{-5}$ & $1.023 \times 10^{-5}$ & $1.055 \times 10^{-3}$ & $1.529 \times 10^{-5}$ & $4.390 \times 10^{-6}$ \\
\hline & OPTE & $1.184 \times 10^{-3}$ & $2.887 \times 10^{-5}$ & $9.878 \times 10^{-6}$ & $1.076 \times 10^{-3}$ & $2.215 \times 10^{-5}$ & $5.103 \times 10^{-6}$ \\
\hline \multirow[t]{4}{*}{$\mathrm{CO}$} & CMO & $3.445 \times 10^{-2}$ & $3.408 \times 10^{-3}$ & $5.631 \times 10^{-5}$ & $3.430 \times 10^{-2}$ & $3.397 \times 10^{-3}$ & $5.130 \times 10^{-5}$ \\
\hline & NO & $1.022 \times 10^{-1}$ & $3.337 \times 10^{-2}$ & $8.344 \times 10^{-4}$ & $1.020 \times 10^{-1}$ & $3.336 \times 10^{-2}$ & $8.298 \times 10^{-4}$ \\
\hline & $\mathbf{M}_{\min }$ & $3.599 \times 10^{-2}$ & $4.224 \times 10^{-3}$ & $1.960 \times 10^{-4}$ & $3.576 \times 10^{-2}$ & $4.213 \times 10^{-3}$ & $1.913 \times 10^{-4}$ \\
\hline & OPTE & $1.112 \times 10^{-2}$ & $1.107 \times 10^{-3}$ & $2.084 \times 10^{-5}$ & $1.082 \times 10^{-2}$ & $1.098 \times 10^{-3}$ & $1.599 \times 10^{-5}$ \\
\hline \multirow[t]{4}{*}{$\mathrm{CN}^{-}$} & $\mathrm{CMO}$ & $9.403 \times 10^{-2}$ & $1.139 \times 10^{-2}$ & $9.712 \times 10^{-4}$ & $9.395 \times 10^{-2}$ & $1.138 \times 10^{-2}$ & $9.666 \times 10^{-4}$ \\
\hline & NO & $1.448 \times 10^{-2}$ & $5.759 \times 10^{-4}$ & $2.394 \times 10^{-5}$ & $1.435 \times 10^{-2}$ & $5.668 \times 10^{-4}$ & $1.957 \times 10^{-5}$ \\
\hline & $\mathrm{M}_{\min }$ & $9.673 \times 10^{-3}$ & $3.410 \times 10^{-4}$ & $1.632 \times 10^{-5}$ & $9.583 \times 10^{-3}$ & $3.271 \times 10^{-4}$ & $1.167 \times 10^{-5}$ \\
\hline & OPTE & $3.779 \times 10^{-3}$ & $1.813 \times 10^{-4}$ & $1.042 \times 10^{-5}$ & $3.617 \times 10^{-3}$ & $1.750 \times 10^{-4}$ & $6.266 \times 10^{-6}$ \\
\hline \multirow[t]{4}{*}{$\mathrm{NO}^{+}$} & $\mathrm{CMO}$ & $1.050 \times 10^{-1}$ & $1.188 \times 10^{-2}$ & $1.281 \times 10^{-3}$ & $1.049 \times 10^{-1}$ & $1.187 \times 10^{-2}$ & $1.275 \times 10^{-3}$ \\
\hline & NO & $2.070 \times 10^{-2}$ & $8.607 \times 10^{-4}$ & $3.743 \times 10^{-5}$ & $2.050 \times 10^{-2}$ & $8.510 \times 10^{-4}$ & $3.056 \times 10^{-5}$ \\
\hline & $\mathrm{M}_{\min }$ & $1.318 \times 10^{-2}$ & $5.114 \times 10^{-4}$ & $2.221 \times 10^{-5}$ & $1.300 \times 10^{-2}$ & $5.039 \times 10^{-4}$ & $1.513 \times 10^{-5}$ \\
\hline & OPTE & $4.237 \times 10^{-3}$ & $2.527 \times 10^{-4}$ & $1.317 \times 10^{-5}$ & $3.999 \times 10^{-3}$ & $2.375 \times 10^{-4}$ & $6.327 \times 10^{-6}$ \\
\hline
\end{tabular}

$$
\begin{aligned}
t_{p q} & =\langle p|h| q\rangle, \\
V_{p q r s} & =\langle p q|v| r s\rangle,
\end{aligned}
$$

where $\langle p|h| q\rangle$ and $\langle p q|v| r s\rangle$ are the one- and two-electron antisymmetrized integrals, respectively, expressed in the Dirac notation. In this case, the DOCI Hamiltonian (14) reduces to

$$
H_{\mathrm{mol}}=2 \sum_{i} t_{i i} n_{i}+\sum_{i \neq j}\left(2 V_{i j i j}-V_{i j j i}\right) n_{i} n_{j}+\sum_{i j} V_{i i j j} b_{i}^{\dagger} b_{j} .
$$

We have for the first time investigated the set of isoelectronic diatomic systems $\mathrm{N}_{2}, \mathrm{CO}, \mathrm{CN}^{-}$, and $\mathrm{NO}^{+}$within the full 3POS v2RDM-DOCI theory, determining their ground-state potential energy curves for varying internuclear distances. In these molecular systems, the static correlation is of paramount importance especially at dissociation. The STO-3G, 3-21G, and $\mathrm{DZ}$ atomic basis sets have been used to obtain all the reported results. Given that the DOCI and v2RDM-DOCI results are not invariant with respect to unitary transformation of the basis set used, ${ }^{8,11,31,39,40}$ we have studied their dependence for several basis set choices. In particular, we have performed calculations using the following orthonormal basis sets: the canonical molecular orbitals (CMO), the natural orbitals (NO), the orbitals which minimize the seniority number of the exact FCI expansions $\left(\mathrm{M}_{\min }\right),{ }^{11}$ and the orbitals which minimize the DOCI energy (OPTE) ${ }^{56}$ The one- and two-electron integrals expressed in the CMO basis sets have been computed with the PSI3.4 package. ${ }^{57}$ We have used our own codes to build and diagonalize either the full Hamiltonian (1) to obtain the $\mathrm{NO}$ and $\mathrm{M}_{\min }$ basis sets, or its projection in the seniority-zero space (14) to obtain the
OPTE ones. The calculation of energies and RDMs within DOCI has been implemented using modified versions of the direct determinant-FCI algorithm reported in Refs. 58 and 59.

In Table I, we report the maximum absolute errors (MAE) found between the DOCI energy and those obtained from the use of different $N$-representability constraint sets in the variational method as functions of the internuclear distance, using several orbital basis sets. The values arising

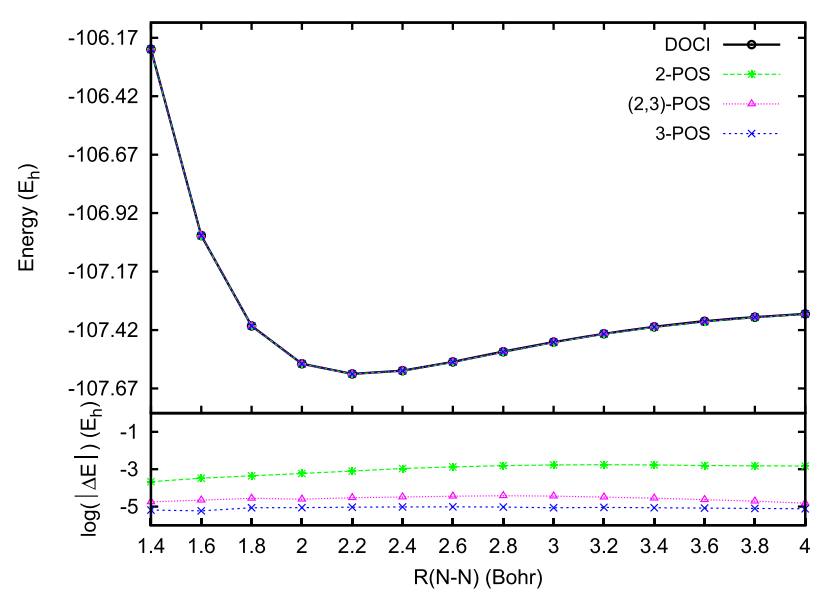

FIG. 1. Ground-state potential energy curve of the $\mathrm{N}_{2}$ molecule calculated by the DOCI and v2RDM-DOCI procedures imposing the 2-POS, (2,3)-POS, and 3-POS conditions. Results correspond to canonical molecular orbitals arising from the STO-3G atomic basis set. The bottom panel shows the logarithm of the absolute values of the energy errors, $|\Delta \mathrm{E}|$, of the v2RDM-DOCI results with respect to the DOCI ones. 


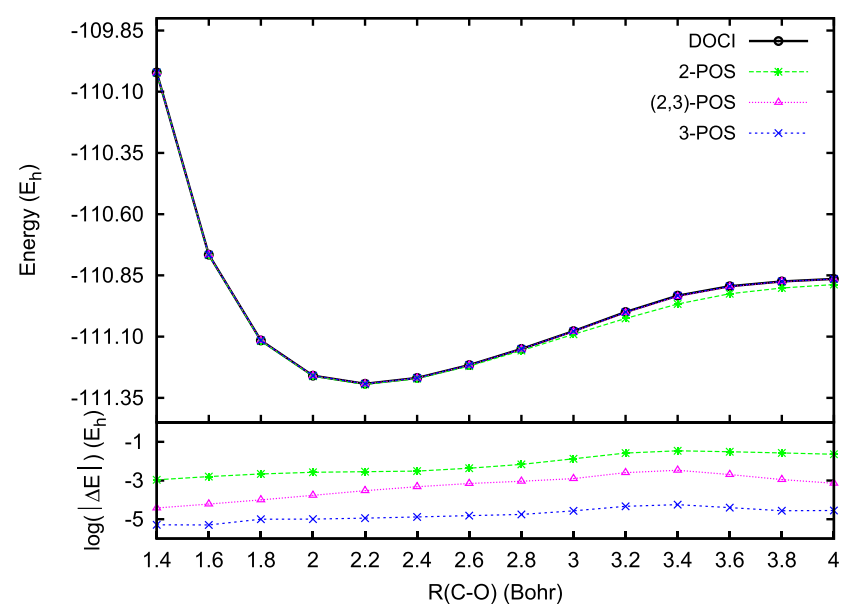

FIG. 2. Ground-state potential energy curve of $\mathrm{CO}$ molecule calculated by the DOCI and v2RDM-DOCI procedures imposing the 2-POS, (2,3)-POS, and 3-POS conditions. Results correspond to canonical molecular orbitals arising from the STO-3G atomic basis set. The bottom panel shows the logarithm of the absolute values of the energy errors, $\mid \Delta \mathrm{El}$, of the v2RDM-DOCI results with respect to the DOCI ones.

from the 2-POS and (2,3)-POS v2RDM-DOCI procedures have been previously reported by us in Ref. 33; they have been included in this and other Tables in order to make an easier comparison with the results obtained from the method proposed in this work. As can be seen, in all cases, the full 3-POS conditions improve the energies from those using the 2- and (2,3)-POS by at least three and two orders of magnitude, respectively. Table I also shows the non-parallelity errors (NPE) in that internuclear distance interval, that is, the differences between the maximum and minimum deviation of the v2RDM-DOCI energy values from the DOCI ones. Both MAE and NPE quantities have very similar numerical values, predicting similar behavior. Figures $1-4$ show the potential energy curves obtained with the CMO basis sets for the studied systems, as well as the logarithm of the absolute values

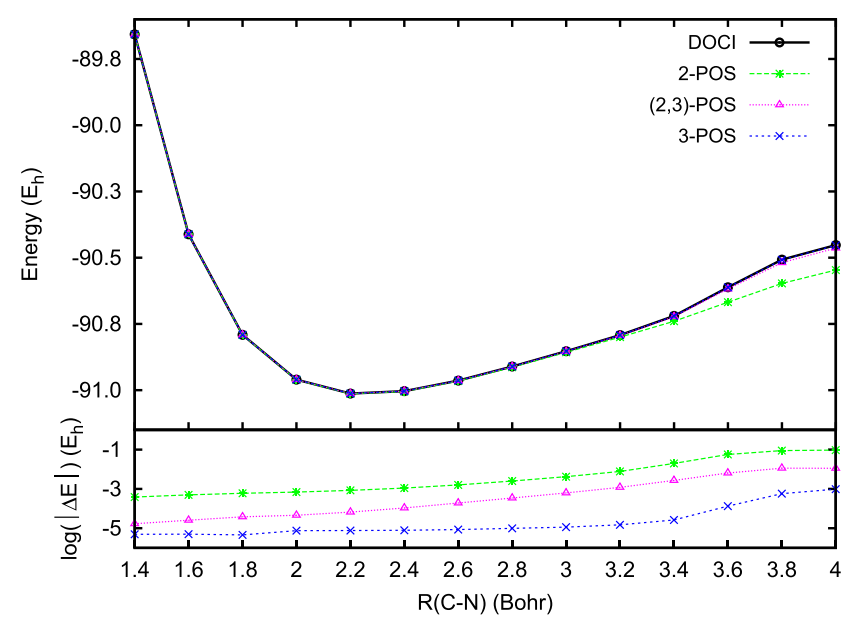

FIG. 3. Ground state potential energy curve of the $\mathrm{CN}^{-}$molecule calculated by the DOCI and V2RDM-DOCI procedures imposing the 2-POS, (2,3)-POS, and 3-POS conditions. Results correspond to canonical molecular orbitals arising from the STO-3G atomic basis set. The bottom panel shows the logarithm of the absolute values of the energy errors, $|\Delta \mathrm{E}|$, of the v2RDM-DOCI results with respect to the DOCI ones.

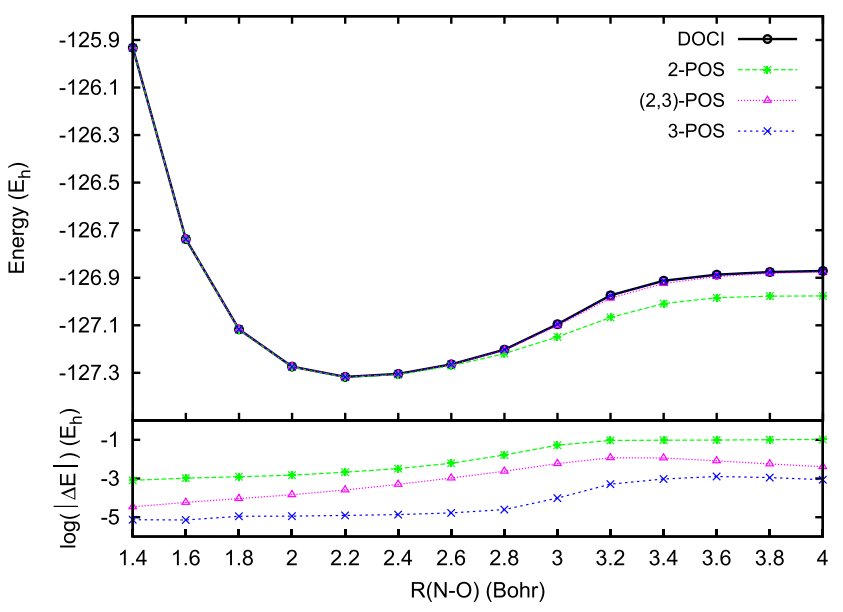

FIG. 4. Ground state potential energy curve of $\mathrm{NO}^{+}$molecule calculated by the DOCI and v2RDM-DOCI procedures imposing the 2-POS, (2,3)-POS, and 3-POS conditions. Results correspond to canonical molecular orbitals arising from the STO-3G atomic basis set. The bottom panel shows the logarithm of the absolute values of the energy errors, $|\triangle \mathrm{E}|$, of the v2RDM-DOCI results with respect to the DOCI ones.

of the energy errors, $\mid \Delta \mathrm{El}$, relative to the corresponding reference DOCI values. These results demonstrate that the 3-POS variational method yields the closest values to the DOCI one, in agreement with those exhibited in Table I. The variational lower bound 3-POS v2RDM-DOCI curve is essentially indistinguishable from the DOCI curve, although its values always lie below the DOCI ones. Therefore, it is worth noting that the 3-POS conditions strongly reduce the 2- and $(2,3)$-POS non-parallelity errors near bond dissociation. Moreover, the comparative analysis of the counterpart values arising from the different molecular orbital basis sets, reported in Table I, shows that the OPTE one leads to the most accurate results, the $\mathrm{M}_{\min }$ basis set presents better behavior than the natural orbitals, while the CMO basis set provides a poor approximation. These results are illustrated in Fig. 5, which shows

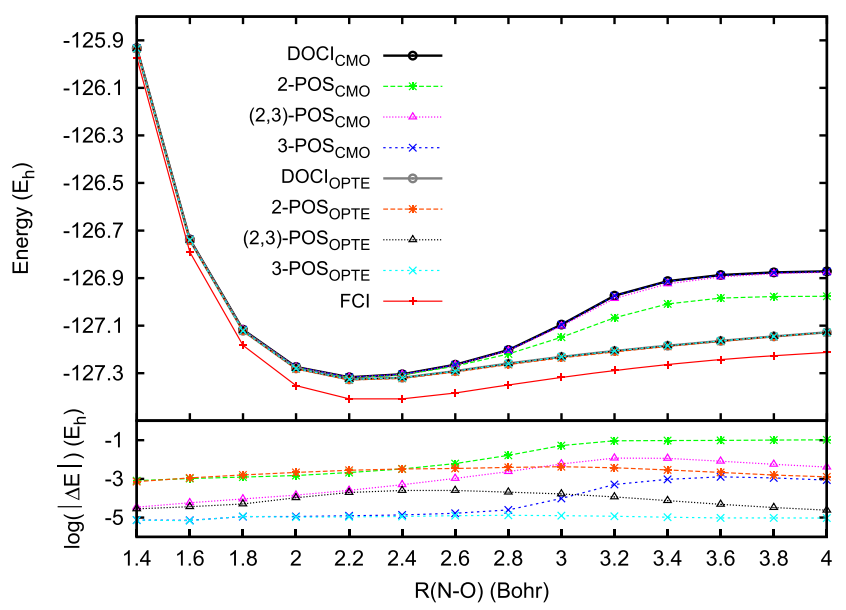

FIG. 5. Ground state potential energy curve of $\mathrm{NO}^{+}$system calculated by the FCI, DOCI, and v2RDM-DOCI procedures imposing the 2-POS, (2,3)-POS, and 3-POS conditions, in the CMO and in the OPTE molecular orbitals arising from the STO-3G atomic basis set. The bottom panel shows the logarithm of the absolute values of the energy errors, $|\Delta \mathrm{E}|$, of the v2RDM-DOCI results with respect to the DOCI ones. 
TABLE II. Energy errors in the ground state of the $\mathrm{NO}^{+}$molecule at two bond lengths calculated in the canonical molecular orbitals (CMO), natural orbitals (NO), orbitals which minimize the seniority number of the FCI wave functions $\left(\mathrm{M}_{\min }\right)$, and orbitals which minimize the DOCI energy (OPTE). The errors calculated by the DOCI variational RDM method (v2DM-DOCI) are computed imposing the 2-POS, (2,3)-POS, and 3-POS conditions. Results correspond to standard STO-3G basis set. The 2-POS and (2,3)-POS data have already been described in Ref. 33 .

\begin{tabular}{|c|c|c|c|c|}
\hline & \multirow[b]{2}{*}{$\mathrm{E}_{\mathrm{DOCI}}\left(E_{h}\right)$} & \multicolumn{3}{|c|}{$\mathrm{E}_{\mathrm{v} 2 \mathrm{RDM}-\mathrm{DOCI}}-\mathrm{E}_{\mathrm{DOCI}}\left(E_{h}\right)$} \\
\hline & & 2-POS & $(2,3)-\mathrm{POS}$ & 3-POS \\
\hline & \multicolumn{4}{|c|}{$\mathrm{R}_{\mathrm{eq}}=2.2 \mathrm{bohr}$} \\
\hline $\mathrm{CMO}$ & -127.316934 & $-2.121 \times 10^{-3}$ & $-2.542 \times 10^{-4}$ & $-1.239 \times 10^{-5}$ \\
\hline NO & -127.320704 & $-1.985 \times 10^{-3}$ & $-1.681 \times 10^{-4}$ & $-1.080 \times 10^{-5}$ \\
\hline $\mathrm{M}_{\min }$ & -127.324924 & $-3.901 \times 10^{-3}$ & $-2.658 \times 10^{-4}$ & $-1.127 \times 10^{-5}$ \\
\hline \multirow[t]{2}{*}{ OPTE } & -127.326027 & $-2.795 \times 10^{-3}$ & $-1.980 \times 10^{-4}$ & $-1.082 \times 10^{-5}$ \\
\hline & \multicolumn{4}{|c|}{$\mathrm{R}_{\mathrm{st}}=4.0 \mathrm{bohr}$} \\
\hline $\mathrm{CMO}$ & -126.871538 & $-1.050 \times 10^{-1}$ & $-4.068 \times 10^{-3}$ & $-8.673 \times 10^{-4}$ \\
\hline NO & -127.119269 & $-1.795 \times 10^{-2}$ & $-4.600 \times 10^{-4}$ & $-2.796 \times 10^{-5}$ \\
\hline $\mathrm{M}_{\min }$ & -127.121756 & $-1.236 \times 10^{-2}$ & $-2.584 \times 10^{-4}$ & $-1.870 \times 10^{-5}$ \\
\hline OPTE & -127.128085 & $-1.276 \times 10^{-3}$ & $-2.415 \times 10^{-5}$ & $-9.483 \times 10^{-6}$ \\
\hline
\end{tabular}

the potential energy curves for 2-, (2,3)-, and 3-POS-v2RDMDOCI methods, as well as for the DOCI and FCI ones, for the $\mathrm{NO}^{+}$system, in the CMO and OPTE molecular basis sets.

We have gathered in Table II the values of the $\mathrm{NO}^{+}$energy differences $E_{\mathrm{V} 2 \mathrm{RDM}}$-DOCI $-\mathrm{E}_{\mathrm{DOCI}}$ obtained for each of the above-mentioned orthonormal basis sets by imposing 2-, (2,3), and 3-POS $N$-representability conditions. These values have been obtained at both an internuclear distance near the equilibrium (2.2 bohr) and at a stretched geometry (4.0 bohr). These results again show a clear improvement according to the sequence of imposed conditions 2-POS, (2,3)-POS, and 3-POS as well as to the basis set sequence $\mathrm{CMO}, \mathrm{NO}, \mathrm{M}_{\min }$, OPTE. To complete this study, we report in Table III values of the root-mean-square deviation of the $\Pi$ and $D$ seniority blocks of ${ }^{2} D_{\text {v2RDM-DOCI }}$ with respect to those of ${ }^{2} D_{\text {DOCI }}$, for the $\mathrm{NO}^{+}$ system at two internuclear distances. As can be observed, these values confirm, in terms of differences of 2-RDM elements, the conclusions arising from the numerical values found in terms of energy differences, showing similar behavior. The 3-POS conditions improve upon the accuracy of the 2-RDMs from 2POS by two-to-three orders of magnitude while they improve upon the accuracy from $(2,3)$-POS by one-half-to-two orders of magnitude.

In order to test the effect on the results of the chosen atomic basis set, we compare in Tables IV and V the energy and 2-RDM differences between $\mathrm{V} 2 \mathrm{RDM}$-DOCI and DOCI for the imposed conditions series 2-POS, (2,3)-POS, and 3-POS and for different basis sets. The results for the energies and their comparison with those reported in Table II indicate that there is no drastic effect of the 3-POS conditions when using the CMO orthonormal basis at the equilibrium geometry. On the other hand, at stretched geometries, the larger basis sets give somewhat smaller energy differences. In Table $\mathrm{V}$, the differences in the 2-RDM and their comparison with those reported in Table III also show that the influence is moderated although in this case the results suggest that there is a small improvement for the larger basis sets.

\section{Pairing models}

Attractive pairing Hamiltonians based on the SU(2) algebra describe the fundamental physics of superconductivity in macroscopic systems of condensed matter, ${ }^{60}$ and neutron stars, ${ }^{61}$ as well as in mesoscopic systems like atomic nuclei, ${ }^{61,62}$ cold atoms, ${ }^{63,64}$ and ultrasmall superconducting

TABLE III. Root-mean-square deviations of the $\Pi$ and $D$ seniority blocks of the 2-RDM with respect to the DOCI ones in the ground state of the $\mathrm{NO}^{+}$molecule at two bond lengths calculated in the canonical molecular orbitals (CMO), natural orbitals (NO), orbitals which minimize the seniority number of the FCI wave functions $\left(\mathrm{M}_{\min }\right)$ and orbitals which minimize the DOCI energy (OPTE). The errors calculated by the DOCI variational RDM method (v2DM-DOCI) are computed imposing the 2-POS, (2,3)-POS, and 3-POS conditions. Results correspond to standard STO-3G basis set.

\begin{tabular}{|c|c|c|c|c|c|c|}
\hline & \multicolumn{6}{|c|}{$\left\|^{2} D_{\mathrm{v} 2 \mathrm{RDM}-\mathrm{DOCI}}-{ }^{2} D_{\mathrm{DOCI}}\right\|$} \\
\hline & \multicolumn{3}{|c|}{$\Pi$} & \multicolumn{3}{|c|}{$D$} \\
\hline & 2-POS & $(2,3)-\mathrm{POS}$ & 3-POS & 2-POS & $(2,3)-\mathrm{POS}$ & 3-POS \\
\hline & \multicolumn{6}{|c|}{$\mathrm{R}_{\mathrm{eq}}=2.2 \mathrm{bohr}$} \\
\hline $\mathrm{CMO}$ & $1.613 \times 10^{-3}$ & $1.747 \times 10^{-4}$ & $6.466 \times 10^{-6}$ & $1.980 \times 10^{-3}$ & $6.424 \times 10^{-4}$ & $5.175 \times 10^{-6}$ \\
\hline NO & $1.392 \times 10^{-3}$ & $1.080 \times 10^{-4}$ & $2.398 \times 10^{-6}$ & $2.505 \times 10^{-3}$ & $5.050 \times 10^{-4}$ & $2.936 \times 10^{-6}$ \\
\hline $\mathrm{M}_{\min }$ & $2.954 \times 10^{-3}$ & $2.266 \times 10^{-4}$ & $3.583 \times 10^{-6}$ & $2.477 \times 10^{-3}$ & $3.978 \times 10^{-4}$ & $2.073 \times 10^{-6}$ \\
\hline \multirow[t]{2}{*}{ OPTE } & $1.887 \times 10^{-3}$ & $1.821 \times 10^{-4}$ & $3.227 \times 10^{-6}$ & $2.362 \times 10^{-3}$ & $4.358 \times 10^{-4}$ & $2.258 \times 10^{-6}$ \\
\hline & \multicolumn{6}{|c|}{$\mathrm{R}_{\mathrm{st}}=4.0 \mathrm{bohr}$} \\
\hline $\mathrm{CMO}$ & $9.847 \times 10^{-2}$ & $7.621 \times 10^{-3}$ & $3.400 \times 10^{-3}$ & $1.337 \times 10^{-1}$ & $1.190 \times 10^{-2}$ & $3.337 \times 10^{-3}$ \\
\hline NO & $1.150 \times 10^{-2}$ & $5.596 \times 10^{-4}$ & $1.364 \times 10^{-4}$ & $5.504 \times 10^{-2}$ & $1.069 \times 10^{-3}$ & $4.889 \times 10^{-5}$ \\
\hline $\mathrm{M}_{\min }$ & $9.848 \times 10^{-3}$ & $4.163 \times 10^{-4}$ & $4.507 \times 10^{-5}$ & $5.347 \times 10^{-2}$ & $7.817 \times 10^{-4}$ & $3.314 \times 10^{-5}$ \\
\hline OPTE & $2.768 \times 10^{-2}$ & $2.595 \times 10^{-4}$ & $6.366 \times 10^{-5}$ & $4.075 \times 10^{-2}$ & $1.569 \times 10^{-4}$ & $6.674 \times 10^{-6}$ \\
\hline
\end{tabular}


TABLE IV. Energy errors in the ground state of the $\mathrm{NO}^{+}$molecule at two bond lengths calculated in the canonical molecular orbitals (CMO). The errors calculated by the DOCI variational RDM method (v2RDM-DOCI) are computed imposing the 2-POS, (2,3)-POS, and 3-POS conditions. Results were obtained using the 3-21G and DZ atomic basis sets. The 2-POS and (2,3)-POS data have already been described in Ref. 33 .

\begin{tabular}{lcccc}
\hline & & \multicolumn{4}{c}{$\mathrm{E}_{\mathrm{v} 2 \mathrm{RDM}-\mathrm{DOCI}}-\mathrm{E}_{\mathrm{DOCI}}\left(E_{h}\right)$} \\
\cline { 3 - 5 } & $\mathrm{E}_{\mathrm{DOCI}}\left(E_{h}\right)$ & 2 2-POS & $(2,3)-\mathrm{POS}$ & $3-\mathrm{POS}$ \\
\hline \multicolumn{5}{c}{$\mathrm{R}_{\mathrm{eq}}=2.2 \mathrm{bohr}$} \\
\hline 3-21G & -128.210830 & $-3.565 \times 10^{-3}$ & $-3.538 \times 10^{-4}$ & $-1.921 \times 10^{-5}$ \\
DZ & -128.914167 & $-2.448 \times 10^{-3}$ & $-3.374 \times 10^{-4}$ & $-2.544 \times 10^{-5}$ \\
\hline & & \multicolumn{4}{c}{$\mathrm{R}_{\mathrm{st}}=4.0 \mathrm{bohr}$} & \\
\hline $3-21 \mathrm{G}$ & -127.818807 & $-4.652 \times 10^{-2}$ & $-2.924 \times 10^{-3}$ & $-4.531 \times 10^{-4}$ \\
DZ & -128.518533 & $-3.104 \times 10^{-2}$ & $-2.036 \times 10^{-3}$ & $-2.519 \times 10^{-4}$ \\
\hline \hline
\end{tabular}

grains ${ }^{65,66}$ A large class of these Hamiltonians can be derived from the quantum integrable Richardson-Gaudin (RG) pairing models. ${ }^{67,68}$ These models, being exactly solvable for attractive and repulsive interactions, provide ideal Hamiltonians to study the effects of strong correlation and to test the accuracy of approximate methods such as the variational reduced density matrix method within the seniority-zero subspace. Here, we will benchmark the variational method with the hyperbolic RG model leading to separable pairing Hamiltonians. ${ }^{68}$ It has been shown that these Hamiltonians naturally represent systems of spinless fermions with $p$-wave pairing interactions. In $2 \mathrm{D}$, the hyperbolic model gives rise to the $p_{x}+i p_{y}$ model, ${ }^{69,70}$ and in 1D to an interacting and number conserving Kitaev wire. ${ }^{71}$ Both Hamiltonians display a quantum phase transition to a topological superfluid in the attractive branch. On the contrary, the repulsive branch has been related to the physics of quantum Hall systems. ${ }^{72}$ Here we will explore the repulsive interaction side as it is more akin to the physics of quantum chemistry.

The separable pairing Hamiltonian can be derived as a linear combination of the hyperbolic RG integrals of motion

$$
H_{\text {hyp }}=\sum_{i} \varepsilon_{i} n_{i}+G \sum_{i j} \sqrt{\varepsilon_{i} \varepsilon_{j}} b_{i}^{\dagger} b_{j},
$$

where $G$ is the interaction strength and $\varepsilon_{i}$ are the single-particle energies. For simplicity, we will treat half-filled systems with the number of pairs $M=K / 2$, where $K$ is the total number of levels, and with equidistant single-particle energies defined as $\varepsilon_{i}=i / K, i=1,2, \ldots, K$. The eigenstates of the hyperbolic Hamiltonian (40) are

$$
|\Psi\rangle=\prod_{\alpha=1}^{M}\left[\sum_{i=1}^{K} \frac{\sqrt{\varepsilon_{i}}}{\varepsilon_{i}-E_{\alpha}} b_{i}^{\dagger}\right]|0\rangle,
$$

where $|0\rangle$ is the vacuum state. Each eigenstate is determined by $M$ pair energies $E_{\alpha}$ that are a particular solution of a set of $M$ non-linear coupled Richardson equations. ${ }^{68}$ Therefore, the complexity of the model Hamiltonian increases linearly with the number of pairs $M$, and we can look for exact ground-state energies for sizes beyond the limits of an exact diagonalization. Here we consider a system of $K=40$ levels at half-filling corresponding to a dimension of the Hamiltonian matrix of $1.4 \times 10^{11}$. The exact solution is obtained by solving a set of 20 non-linear coupled equations. Alternatively, one can avoid the divergencies that arise when solving the $M$ Richardson equations by using the eigenvalue-based method of Ref. 73 that reduces the solution to a set of $K$ quadratic equations. In both cases, the complexity of the integrable model increases linearly with the system size.

Figure 6 compares the energy logarithmic errors of the v2RDM-DOCI procedures imposing 2-POS, (2,3)-POS, and 3-POS conditions as a function repulsive pairing strength $G$. We consider a quite large interval of the interaction strength going up to twice the bandwidth $(G=2.0)$. The left inset displays the ground-state energy as a function of $G$ with two marked regimes. At weak coupling, the system behaves as a normal metal, while at stronger couplings it resembles a strongly correlated Fermi liquid. Both characteristics can be seen in the right inset where we plot the occupation probabilities $\rho_{i}$ for $G=0$ and $G=2.0$ evaluated from the exact solution. The errors in the ground-state energy for the 2-POS, the $(2,3)$ POS, and the 3-POS follow a similar pattern. Once the Fermi

TABLE V. Root-mean-square deviations of the $\Pi$ and $D$ seniority blocks of the 2-RDM with respect to the DOCI ones in the $\mathrm{NO}^{+}$molecule at two bond lengths, calculated for the v2RDM-DOCI method in the canonical molecular orbitals (CMO). The errors are computed imposing the 2-POS, (2,3)-POS, and 3-POS conditions. Results were

\begin{tabular}{|c|c|c|c|c|c|c|}
\hline & \multicolumn{6}{|c|}{$\left\|^{2} D_{\mathrm{V} 2 \mathrm{RDM}-\mathrm{DOCI}}-{ }^{2} D_{\mathrm{DOCI}}\right\|$} \\
\hline & \multicolumn{3}{|c|}{$\Pi$} & \multicolumn{3}{|c|}{$D$} \\
\hline & 2-POS & $(2,3)-\mathrm{POS}$ & 3-POS & 2-POS & $(2,3)-\mathrm{POS}$ & 3-POS \\
\hline & \multicolumn{6}{|c|}{$\mathrm{R}_{\mathrm{eq}}=2.2 \mathrm{bohr}$} \\
\hline $3-21 G$ & $1.121 \times 10^{-3}$ & $1.088 \times 10^{-4}$ & $3.742 \times 10^{-6}$ & $9.917 \times 10^{-4}$ & $3.014 \times 10^{-4}$ & $4.403 \times 10^{-6}$ \\
\hline \multirow[t]{2}{*}{$\mathrm{DZ}$} & $7.738 \times 10^{-4}$ & $1.070 \times 10^{-4}$ & $4.470 \times 10^{-6}$ & $5.639 \times 10^{-4}$ & $2.733 \times 10^{-4}$ & $3.757 \times 10^{-6}$ \\
\hline & \multicolumn{6}{|c|}{$\mathrm{R}_{\mathrm{st}}=4.0 \mathrm{bohr}$} \\
\hline $3-21 G$ & $4.743 \times 10^{-2}$ & $2.829 \times 10^{-3}$ & $7.299 \times 10^{-4}$ & $6.147 \times 10^{-2}$ & $2.304 \times 10^{-3}$ & $1.717 \times 10^{-3}$ \\
\hline $\mathrm{DZ}$ & $3.764 \times 10^{-2}$ & $1.891 \times 10^{-3}$ & $3.185 \times 10^{-4}$ & $4.412 \times 10^{-2}$ & $1.616 \times 10^{-3}$ & $6.599 \times 10^{-4}$ \\
\hline
\end{tabular}
obtained using the $3-21 \mathrm{G}$ and $\mathrm{DZ}$ atomic basis sets. 


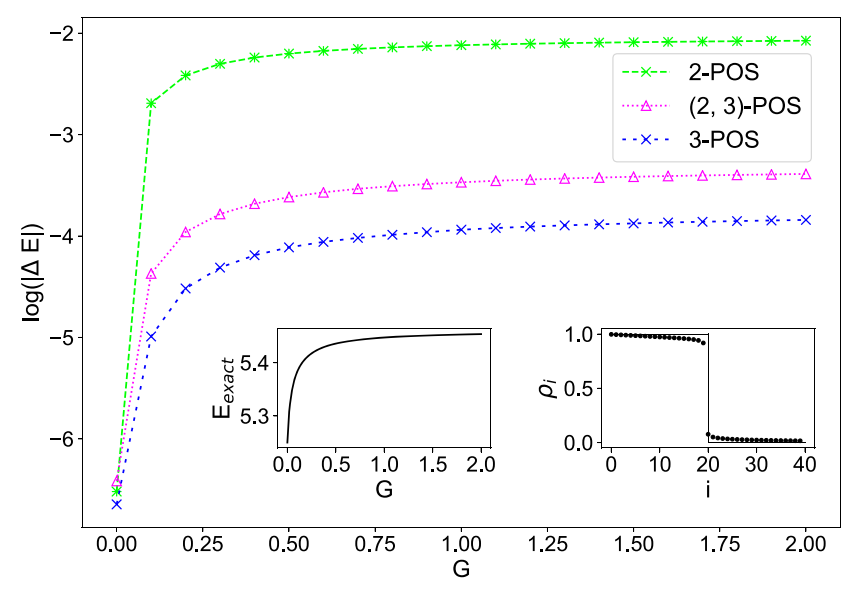

FIG. 6. Logarithm of the absolute values of energy errors, $|\Delta E|$, for the ground-state as a function of the coupling $G$ for the repulsive separable pairing Hamiltonian with $K=2 M=40$ calculated within v2RDM-DOCI imposing the 2-POS, the (2,3)-POS and the 3-POS conditions. The left inset shows the ground-state energy as a function of $G$ and the right inset displays the occupation probabilities $\rho_{i}$ for the two extreme cases: $G=0$ (continuous line) and $G=2$ (filled circles).

liquid is stabilized, the accuracy of the (2,3)-POS conditions improves one order of magnitude over the 2-POS conditions, and the complete 3-POS conditions gain an additional 3-4 factor. It is interesting to point out that while the $(2,3)$-POS conditions are sufficient to obtain the exact ground-state energy for attractive pairing Hamiltonians, ${ }^{35}$ they are not enough for the same Hamiltonians with repulsive interactions despite the high accuracy $\left(\sim 10^{-5}\right)$ in the relative ground-state energy error. The reason for the different behavior of the $\mathrm{v} 2 \mathrm{RDM}$-DOCI between attractive and repulsive pairing interactions is still an open and intriguing question.

\section{SUMMARY AND CONCLUSIONS}

In this work, we have studied the effect of the full threeparticle $N$-representability conditions in the variational determination of the two-particle reduced density matrix within the seniority-zero subspace. These conditions constitute necessary constraints to ensure the $N$-representability of the two-particle reduced density matrix. We have focused on a set of isoelectronic molecules and exactly solvable pairing models with repulsive interactions. The results obtained show an important improvement upon those obtained using two- and partial-threeparticle $N$-representability conditions, approaching those provided by the exact DOCI method. Moreover, this achievement does not require any (significant) increase of the computational cost. The quality of these numerical determinations evinces the usefulness of the three-particle variational constraints within the doubly occupied configuration interaction framework.

\section{ACKNOWLEDGMENTS}

The authors acknowledge financial support from the Universidad de Buenos Aires (Grant No. 20020150100157BA), the Consejo Nacional de Investigaciones Científicas y Técnicas (Grant Nos. PIP 11220130100377CO, PIP 11220130100311CO, 11220150100442CO, and 2013-1401PCB), the Agencia Nacional de Promoción Científica y Tecnológica, Argentina (Grant No. PICT-201-0381), and the Spanish Ministerio de Economía y Competitividad and the European regional development fund (FEDER) (No. FIS201563770-P).

\section{APPENDIX: TWO- AND PARTIAL THREE-PARTICLE $N$-REPRESENTABILITY CONDITIONS ON THE 2-RDM}

As mentioned in Sec. II, restricting the operator $B$ in Eq. (5) to the two-particle space leads to the $2-\mathcal{P}, 2-\mathcal{Q}$, and $2-\mathcal{G}$ conditions. In the seniority-zero subspace, the 1- and 2-RDM become block diagonal in the seniority number and these conditions can be reformulated in terms of their seniority blocks $\rho, \Pi$, and $D$. Thus, it follows that ${ }^{5,15,31,32,35,39,54}$

- The 2-P condition:

$$
\begin{aligned}
\Pi & \geq 0 \\
D_{i j} & \geq 0, \quad \forall i, j .
\end{aligned}
$$

- The 2- $\mathcal{Q}$ condition:

$$
\begin{aligned}
Q & \geq 0 \\
D_{i j}+1-\rho_{i}-\rho_{j} & \geq 0, \quad \forall i, j,
\end{aligned}
$$

where

$$
Q_{i j}=\Pi_{i j}+\delta_{i j}\left(1-2 \rho_{i}\right)
$$

- The 2-G condition:

$$
\begin{aligned}
G_{i j} & =\left(\begin{array}{cc}
\rho_{i}-D_{i j} & -\Pi_{i j} \\
-\prod_{j i} & \rho_{j}-D_{i j}
\end{array}\right) \geq 0, \quad \forall i>j, \\
g & \geq 0,
\end{aligned}
$$

where

$$
g_{i j}=D_{i j}
$$

From the three-positivity conditions, the following subset depending on the 1- and 2-RDM only can be derived.

- The $\mathcal{T}_{1}$ condition:

$$
\begin{gathered}
T_{1}^{i} \geq 0, \quad \forall i, \\
1-\rho_{i}-\rho_{j}-\rho_{k}+D_{i j}+D_{j k}+D_{k i} \geq 0, \quad \forall i>j>k,
\end{gathered}
$$

where

$$
\left(T_{1}\right)_{j k}^{i}=\delta_{j k}\left(1-2 \rho_{j}-\rho_{i}+2 D_{i j}\right)+\Pi_{j k}, \quad \forall j, k \neq i .
$$


- The $\mathcal{T}_{2}$ condition:

$$
T_{i j k}^{2}=\left(\begin{array}{ccc}
\rho_{i}^{i} \geq 0, & \forall i, \\
\Pi_{i k}-D_{i k}+D_{j k} & \Pi_{i k} & \Pi_{i j} \\
\Pi_{i j} & \rho_{k}-D_{j k}-D_{i k}+D_{i j} & \Pi_{j k} \\
\Pi_{j k} & \rho_{j}-D_{i j}-D_{j k}+D_{i k}
\end{array}\right) \geq 0, \quad \forall i<j<k .
$$

where

$$
T_{2}^{i}=\left(\begin{array}{ccc}
D_{j k} & -\delta_{j k} \Pi_{i k} & D_{i k} \\
-\delta_{j k} \Pi_{k i} & \delta_{j k}\left(\rho_{i}-2 D_{i k}\right)+\Pi_{j k} & \Pi_{i k} \\
D_{j i} & \Pi_{j i} & \rho_{i}
\end{array}\right) .
$$

${ }^{1}$ W. Kohn, in Nobel Lecture Chemistry, edited by I. Grethe (World Scientific, Singapore, 1996-2000; 2003), pp. 213-237.

${ }^{2}$ T. L. Allen and H. Shull, J. Phys. Chem. 66, 2281 (1962).

${ }^{3}$ D. W. Smith and S. J. Fogel, J. Chem. Phys. 43, S91 (1965).

${ }^{4}$ A. Veillard and E. Clementi, Theor. Chim. Acta 7, 133 (1967).

${ }^{5}$ F. Weinhold and E. B. Wilson, J. Chem. Phys. 46, 2752 (1967).

${ }^{6}$ M. Couty and M. B. Hall, J. Phys. Chem. A 101, 6936 (1997).

${ }^{7}$ C. Kollmar and B. A. Heß, J. Chem. Phys. 119, 4655 (2003).

${ }^{8}$ L. Bytautas, T. M. Henderson, C. A. Jiménez-Hoyos, J. K. Ellis, and G. E. Scuseria, J. Chem. Phys. 135, 044119 (2011).

${ }^{9}$ J. M. Wahlen-Strothman, T. M. Henderson, and G. E. Scuseria, Mol. Phys. 116, 186 (2018).

${ }^{10}$ T. Perez and P. Cassam-Chenaï, J. Math. Chem. 56, 1428 (2018).

${ }^{11}$ D. R. Alcoba, A. Torre, L. Lain, G. E. Massaccesi, and O. B. Oña, J. Chem. Phys. 139, 084103 (2013).

${ }^{12}$ P. A. Limacher, P. W. Ayers, P. A. Johnson, S. De Baerdemacker, D. Van Neck, and P. Bultinck, J. Chem. Theory Comput. 9, 1394 (2013).

${ }^{13}$ D. R. Alcoba, A. Torre, L. Lain, G. E. Massaccesi, and O. B. Oña, J. Chem. Phys. 140, 234103 (2014).

${ }^{14}$ D. R. Alcoba, A. Torre, L. Lain, O. B. Oña, P. Capuzzi, M. Van Raemdonck, P. Bultinck, and D. Van Neck, J. Chem. Phys. 141, 244118 (2014).

${ }^{15}$ D. R. Alcoba, A. Torre, L. Lain, O. B. Oña, G. E. Massaccesi, and P. Capuzzi, Advances in Quantum Chemistry (Academic Press, 2018), pp. 315-332.

${ }^{16}$ K. Husimi, Proc. Phys. Math. Soc. Jpn. 22, 264 (1940).

${ }^{17}$ P.-O. Löwdin, Phys. Rev. 97, 1474 (1955).

${ }^{18}$ C. A. Coulson, Rev. Mod. Phys. 32, 170 (1960).

${ }^{19}$ E. R. Davidson, Reduced Density Matrices in Quantum Chemistry (Academic Press, New York, 1976).

${ }^{20}$ A. J. Coleman and V. I. Yukalov, Reduced Density Matrices: Coulson's Challenge, Lecture Notes in Chemistry (Springer Berlin Heidelberg, 2000), p. 282.

${ }^{21}$ D. A. Mazziotti, Reduced-Density-Matrix Mechanics: With Application to Many-Electron Atoms and Molecules, Volume 134 of Advances in Chemical Physics, edited by D. A. Mazziotti (John Wiley \& Sons, Inc., Hoboken, NJ, USA, 2007), pp. 21-59.

${ }^{22}$ E. S. Kryachko and E. V. Ludeña, Phys. Rep. 544, 123 (2014).

${ }^{23}$ R. H. Tredgold, Phys. Rev. 105, 1421 (1957).

${ }^{24}$ A. J. Coleman, Rev. Mod. Phys. 35, 668 (1963).

${ }^{25}$ C. Garrod and J. K. Percus, J. Math. Phys. 5, 1756 (1964).

${ }^{26}$ H. Kummer, J. Math. Phys. 8, 2063 (1967).

${ }^{27}$ A. J. Coleman, Int. J. Quantum Chem. 11, 907 (1977).

${ }^{28}$ D. A. Mazziotti, Phys. Rev. Lett. 108, 263002 (2012).

${ }^{29}$ K. Naftchi-Ardebili, N. W. Hau, and D. A. Mazziotti, Phys. Rev. A 84, 052506 (2011)

${ }^{30}$ D. A. Mazziotti and R. M. Erdahl, Phys. Rev. A 63, 042113 (2001).

${ }^{31}$ W. Poelmans, M. Van Raemdonck, B. Verstichel, S. De Baerdemacker, A. Torre, L. Lain, G. E. Massaccesi, D. R. Alcoba, P. Bultinck, and D. Van Neck, J. Chem. Theory Comput. 11, 4064 (2015).

${ }^{32}$ K. Head-Marsden and D. A. Mazziotti, J. Chem. Phys. 147, 084101 (2017).

${ }^{33}$ D. R. Alcoba, A. Torre, L. Lain, G. E. Massaccesi, O. B. Oña, E. M. Honoré, W. Poelmans, D. Van Neck, P. Bultinck, and S. De Baerdemacker, J. Chem. Phys. 148, 024105 (2018).

${ }^{34}$ B. Verstichel, H. van Aggelen, D. V. Neck, P. Bultinck, S. D. Baerdemacker, D. Van Neck, P. Bultinck, and S. De Baerdemacker, Comput. Phys. Commun. 182, 1235 (2011).
${ }^{35}$ A. Rubio-García, D. R. Alcoba, P. Capuzzi, and J. Dukelsky, J. Chem. Theory Comput. 14, 4183 (2018)

${ }^{36}$ C. Lanssens, P. W. Ayers, D. Van Neck, S. De Baerdemacker, K. Gunst, and P. Bultinck, J. Chem. Phys. 148, 084104 (2018).

${ }^{37}$ M. Yamashita, K. Fujisawa, M. Fukuda, K. Kobayashi, K. Nakata, and M. Nakata, in Semidefinite, Conic and Polynomial Optimization, edited by M. F. Anjos and J. B. Lasserre (Springer, New York, 2011), p. 687.

${ }^{38}$ M. Yamashita, K. Fujisawa, K. Nakata, M. Nakata, M. Fukuda, K. Kobayashi, and K. Goto, "A high-performance software package for semidefinite programs: SDPA 7," Technical Report B-460, Department of Mathematical and Computing Science, Tokyo Institute of Technology, 2010.

${ }^{39}$ W. Poelmans, "Variational determination of the two-particle density matrix: The case of doubly-occupied space," Ph.D. thesis, Ghent University, 2015.

${ }^{40}$ P. A. Limacher, T. D. Kim, P. W. Ayers, P. A. Johnson, S. De Baerdemacker, D. Van Neck, and P. Bultinck, Mol. Phys. 112, 853 (2014).

${ }^{41} \mathrm{P}$. Jorgensen, Second Quantization-Based Methods in Quantum Chemistry (Academic Press, 1981)

${ }^{42}$ D. A. Mazziotti, Phys. Rev. A 65, 062511 (2002).

${ }^{43}$ D. A. Mazziotti, Phys. Rev. A 74, 032501 (2006).

${ }^{44}$ G. Gidofalvi and D. A. Mazziotti, J. Chem. Phys. 126, 024105 (2007).

${ }^{45}$ D. A. Mazziotti, Chem. Rev. 112, 244 (2012).

${ }^{46}$ R. M. Erdahl, Int. J. Quantum Chem. 13, 697 (1978).

${ }^{47}$ Z. Zhao, B. J. Braams, M. Fukuda, M. L. Overton, and J. K. Percus, J. Chem. Phys. 120, 2095 (2004).

${ }^{48}$ D. A. Mazziotti, Phys. Rev. A 72, 032510 (2005).

${ }^{49}$ Y. Nesterov and A. Nemirovskii, Interior-Point Polynomial Algorithms in Convex Programming (Society for Industrial and Applied Mathematics, 1994).

${ }^{50}$ L. Vandenberghe and S. Boyd, SIAM Rev. 38, 49 (1996).

${ }^{51} \mathrm{~S}$. Wright, in Handbook of Semidefinite Programming, 1st ed., International Series in Operations Research and Management Science, edited by H. Wolkowicz, R. Saigal, and L. Vandenberghe (Springer US, 2000), Vol. 27.

${ }^{52}$ S. Wright, Primal-Dual Interior-Point Methods (Society for Industrial and Applied Mathematics, 1997).

${ }^{53}$ I. Talmi, Simple Models of Complex Nuclei (Harwood Academic Publishers, Chur, Switzerland, Langhorne, Pa., USA, 1993).

${ }^{54}$ F. Weinhold and E. B. Wilson, J. Chem. Phys. 47, 2298 (1967).

${ }^{55}$ M. Nakata, B. J. Braams, K. Fujisawa, M. Fukuda, J. K. Percus, M. Yamashita, and Z. Zhao, J. Chem. Phys. 128, 164113 (2008).

${ }^{56}$ M. Van Raemdonck, D. R. Alcoba, W. Poelmans, S. De Baerdemacker, A. Torre, L. Lain, G. E. Massaccesi, D. Van Neck, and P. Bultinck, J. Chem. Phys. 143, 104106 (2015).

${ }^{57}$ T. D. Crawford, C. D. Sherrill, E. F. Valeev, J. T. Fermann, R. A. King, M. L. Leininger, S. T. Brown, C. L. Janssen, E. T. Seidl, J. P. Kenny, and W. D. Allen, J. Comput. Chem. 28, 1610 (2007).

${ }^{58}$ G. Knizia and G. K.-L. Chan, Phys. Rev. Lett. 109, 186404 (2012).

${ }^{59}$ G. Knizia and G. K.-L. Chan, J. Chem. Theory Comput. 9, 1428 (2013).

${ }^{60}$ J. Bardeen, L. N. Cooper, and J. R. Schrieffer, Phys. Rev. 108, 1175 (1957).

${ }^{61}$ D. J. Dean and M. Hjorth-Jensen, Rev. Mod. Phys. 75, 607 (2003).

${ }^{62}$ J. Dukelsky, S. Lerma H., L. M. Robledo, R. Rodriguez-Guzman, and S. M. A. Rombouts, Phys. Rev. C 84, 061301 (2011).

${ }^{63}$ A. Perali, P. Pieri, L. Pisani, and G. C. Strinati, Phys. Rev. Lett. 92, 220404 (2004)

${ }^{64}$ G. Ortiz and J. Dukelsky, Phys. Rev. A 72, 043611 (2005). 
${ }^{65}$ J. von Delft, A. D. Zaikin, D. S. Golubev, and W. Tichy, Phys. Rev. Lett. 77, 3189 (1996).

${ }^{66}$ J. Dukelsky and G. Sierra, Phys. Rev. Lett. 83, 172 (1999).

${ }^{67}$ J. Dukelsky, S. Pittel, and G. Sierra, Rev. Mod. Phys. 76, 643 (2004).

${ }^{68}$ G. Ortiz, R. Somma, J. Dukelsky, and S. Rombouts, Nucl. Phys. B 707, 421 (2005).

${ }^{69}$ M. Ibañez, J. Links, G. Sierra, and S.-Y. Zhao, Phys. Rev. B 79, 180501 (2009).
${ }^{70}$ S. M. A. Rombouts, J. Dukelsky, and G. Ortiz, Phys. Rev. B 82, 224510 (2010).

${ }^{71}$ G. Ortiz, J. Dukelsky, E. Cobanera, C. Esebbag, and C. Beenakker, Phys. Rev. Lett. 113, 267002 (2014).

${ }^{72}$ G. Ortiz, Z. Nussinov, J. Dukelsky, and A. Seidel, Phys. Rev. B 88, 165303 (2013).

${ }^{73}$ P. W. Claeys, S. De Baerdemacker, M. Van Raemdonck, and D. Van Neck, Phys. Rev. B 91, 155102 (2015). 